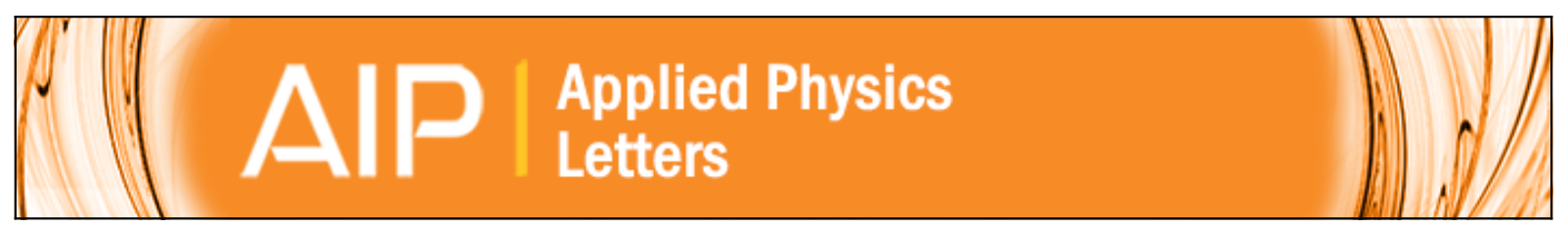

\title{
Memristive operation mode of a site-controlled quantum dot floating gate transistor
}

P. Maier, F. Hartmann, T. Mauder, M. Emmerling, C. Schneider, M. Kamp, S. Höfling, and L. Worschech

Citation: Applied Physics Letters 106, 203501 (2015); doi: 10.1063/1.4921061

View online: http://dx.doi.org/10.1063/1.4921061

View Table of Contents: http://scitation.aip.org/content/aip/journal/apl/106/20?ver=pdfcov

Published by the AIP Publishing

\section{Articles you may be interested in}

Charging dynamics of a floating gate transistor with site-controlled quantum dots

Appl. Phys. Lett. 105, 053502 (2014); 10.1063/1.4892355

Exciton and multiexciton optical properties of single InAs/GaAs site-controlled quantum dots

Appl. Phys. Lett. 103, 183112 (2013); 10.1063/1.4828352

Photocurrent spectroscopy of site-controlled pyramidal quantum dots

Appl. Phys. Lett. 101, 031110 (2012); 10.1063/1.4737426

Single photon emission from site-controlled pyramidal quantum dots

Appl. Phys. Lett. 84, 648 (2004); 10.1063/1.1643533

Optical control of charge number in single floating quantum-dot gate in field-effect transistor structure

Appl. Phys. Lett. 78, 1930 (2001); 10.1063/1.1359139

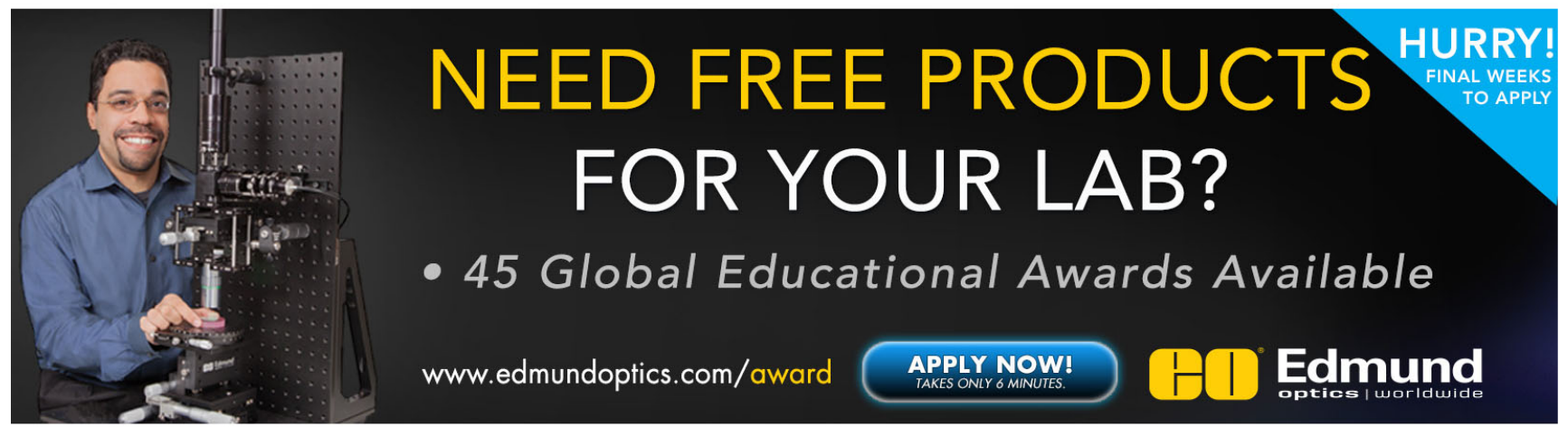




\title{
Memristive operation mode of a site-controlled quantum dot floating gate transistor
}

\author{
P. Maier, ${ }^{1, a)}$ F. Hartmann, ${ }^{1}$ T. Mauder, ${ }^{1}$ M. Emmerling, ${ }^{1}$ C. Schneider, ${ }^{1}$ M. Kamp,${ }^{1}$ \\ S. Höfling, ${ }^{1,2}$ and L. Worschech ${ }^{1}$ \\ ${ }^{1}$ Technische Physik, Physikalisches Institut, Wilhelm Conrad Röntgen Research Center for Complex Material \\ Systems, Universität Würzburg, Am Hubland, D-97074 Würzburg, Germany \\ ${ }_{2}^{2}$ SUPA, School of Physics and Astronomy, University of St Andrews, St Andrews KY16 9SS, United Kingdom
}

(Received 9 March 2015; accepted 2 April 2015; published online 18 May 2015)

\begin{abstract}
We have realized a floating gate transistor based on a GaAs/AlGaAs heterostructure with sitecontrolled InAs quantum dots. By short-circuiting the source contact with the lateral gates and performing closed voltage sweep cycles, we observe a memristive operation mode with pinched hysteresis loops and two clearly distinguishable conductive states. The conductance depends on the quantum dot charge which can be altered in a controllable manner by the voltage value and time interval spent in the charging region. The quantum dot memristor has the potential to realize artificial synapses in a state-of-the-art opto-electronic semiconductor platform by charge localization and Coulomb coupling. (C) 2015 AIP Publishing LLC. [http://dx.doi.org/10.1063/1.4921061]
\end{abstract}

In 1971, Chua postulated the existence of a fourth fundamental circuit element beside the capacitor, inductor, and resistor. $^{1,2}$ This circuit element, now known as memristor, combines the functionality of a switchable resistor (transistor) with memory. Since the pioneering work by Strukov et al. in $2008,{ }^{3}$ which described resistance switching in thin $\mathrm{TiO}_{2}$ layers with oxygen deficient with a memristive model, memristive switching has been identified in various material systems including oxide films, ${ }^{4-6}$ silver compounds, ${ }^{7,8}$ spin memristive systems, ${ }^{9}$ and floating-gate transistors. ${ }^{10,11}$ Memristors are characterized by a pinched hysteresis loop with a state and time dependent resistance or conductance which depends on the previous charge flow through the device. $^{1-3}$ This state-dependent conductance enables memristors to emulate artificially the functionality of synapses, ${ }^{12,13}$ the connections between neurons in neural networks. The strength of these connections can be tuned dynamically depending on the time difference of pre- and post-synaptic pulses, ${ }^{14,15}$ a crucial scheme for learning in neural networks. ${ }^{16,17}$ Artificial synapses are key building blocks in brain-inspired, non-von Neumann architectures with a parallel computing scheme which can for instance be used to implement multiobject detection and classification. ${ }^{18}$

Here, we demonstrate a memristive operation mode of a quantum dot (QD) floating gate transistor by short-circuiting the source contact of the quantum wire $(\mathrm{QW})$ and lateral gates. The memristive operation mode appears due to the Coulomb interaction of QD localized charges with the nearby QW, which leads in one sweep direction of the voltage to a smaller and in the other sweep direction to a larger conductance. We also examined the amount of QD-localized charges which can be controlled via the charging voltage or the time interval spent in the charging region. The memristive operation mode is observed up to temperatures of $165 \mathrm{~K}$. The application of rectangular voltage pulses to the source

\footnotetext{
${ }^{\text {a) }}$ Author to whom correspondence should be addressed. Electronic mail: patrick.maier@physik.uni-wuerzburg.de.
}

and drain contacts of the device allows to tune the conductance in dependence on the time difference of the pulses. This mimics the increase (potentiation) and decrease (depression) of synaptic strength between two neurons by changing the relative timing of pre- and post-synaptic pulses.

Fig. 1(a) depicts an electron microscope image of the studied device. A GaAs/AlGaAs heterostructure was grown by molecular beam epitaxy, forming a two-dimensional electron gas (2DEG) $90 \mathrm{~nm}$ below the surface. Precise positioning of the InAs QDs was realized by a growth step with InAs after defining a regular pattern of $50 \mathrm{~nm}$ deep nanoholes. The whole structure was covered with a GaAs layer. Lateral gates
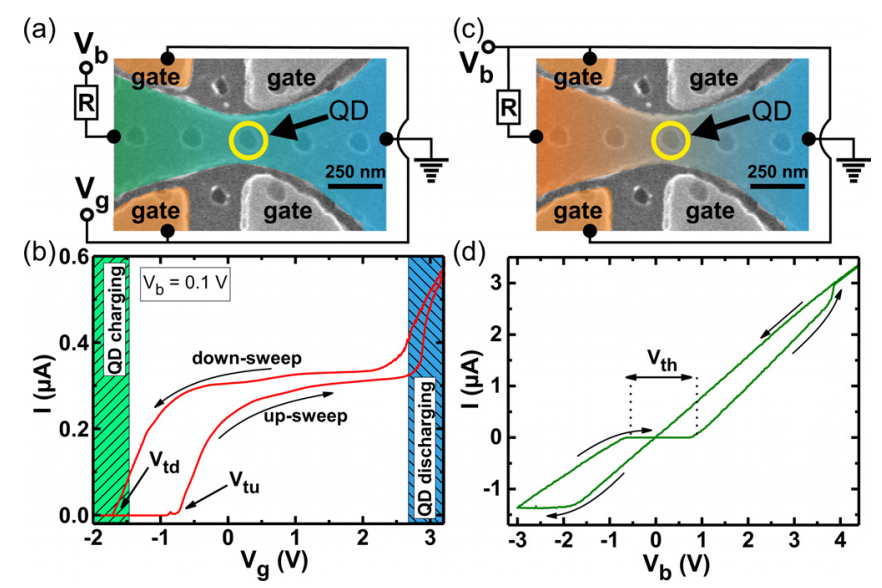

FIG. 1. (a) Electron microscope image of the device and circuit diagram for the floating gate operation mode. The dark contrasts indicate the positions of the QDs and the etched trenches which electrically isolate the quantum wire from the lateral gates. The lateral gates and the QW are connected to the gate voltage $V_{\mathrm{g}}$ and the bias voltage $V_{\mathrm{b}}$, respectively. A yellow circle highlights the QD in the center of the QW. (b) $I-V_{\mathrm{g}}$-characteristic in the floating gate operation mode. The arrows indicate the sweep cycle directions as well as the corresponding threshold voltages $V_{\mathrm{td}}$ and $V_{\mathrm{tu}}$. (c) Circuit diagram to realize the memristive operation mode. The bias voltage $V_{\mathrm{b}}$ is applied simultaneously to the top contact of the QW and the lateral gates. An additional resistance with $1 \mathrm{M} \Omega$ is used in series to the QW. (d) $I-V_{\mathrm{b}}$-characteristic in the memristive operation mode. A pinched hysteresis loop with a width $V_{\text {th }}$ of the low conductive state is observed. 
and a quantum wire structure were realized by electron beam lithography and dry chemical etching. The dark contrast in the electron microscope image indicates the positions of the QDs and the etched trenches to electrically isolate the gates from the QW. A more detailed description of the growth and positioning techniques is given in Refs. 19 and 20.

The device can be operated in two operation modes: the floating gate mode and the memristive mode. Fig. 1(a) displays the circuit diagram in the floating gate operation mode. For this purpose, a constant bias voltage $V_{\mathrm{b}}$ was applied to the left contact of the QW (source) while a gate voltage $V_{\mathrm{g}}$ was applied to the lateral gates. The right contact of the QW (drain) is used as common ground. The current $I$ was determined as voltage drop across a resistor with $10 \mathrm{k} \Omega$ in series to the QW. If not stated differently, the measurements were conducted at $4.2 \mathrm{~K}$ in the dark. Fig. 1 (b) shows the $I-V_{g}$ characteristic of the device in floating gate operation mode. The gate voltage was swept with a rate of $\Delta V_{\mathrm{g}} / \Delta t=0.2 \mathrm{~V} / \mathrm{s}$ from -2.0 to $4.0 \mathrm{~V}$ (up-sweep direction) and back (down-sweep direction), and the bias voltage was set constantly to $V_{\mathrm{b}}=0.1 \mathrm{~V}$. The arrows indicate the gate voltage sweep cycle directions and the corresponding threshold voltages $V_{\mathrm{tu}}$ and $V_{\mathrm{td}}$ for the up- and down-sweep direction, respectively. For small gate voltages, the QW is depleted and the QD becomes charged. $^{20,21}$ After exceeding $V_{\mathrm{tu}}$, the current increases monotonically. $V_{\text {tu }}$ depends on the number of QD localized charges and the capacitive couplings between the gate, the $\mathrm{QD}$, and the QW. ${ }^{22,23}$ The QD becomes discharged for large gate voltages, leading to an enhanced current and a smaller threshold voltage during the down-sweep direction. The approximate gate voltage ranges for charging and discharging the QD are indicated by the shaded regions in Fig. 1(b).

In the memristive operation mode, the bias voltage was applied simultaneously to the source contact of the QW and the lateral gates (see Fig. 1(c)). An additional resistance with $1 \mathrm{M} \Omega$ is connected in series to the channel. The $I-V_{\mathrm{b}}$ characteristic in the memristive operation mode is depicted in Fig. 1(d). The voltage was swept between $4.6 \mathrm{~V}$ and the minimum value $V_{\mathrm{bm}}=-3.0 \mathrm{~V}$. A pinched hysteresis loop, the finger print of memristors, ${ }^{1,2}$ is observed. The differential conductance around zero bias voltage is $0.7 \mu \mathrm{S}$ for the down- and almost zero for the up-sweep direction. Charging of the QD occurs for $V_{\mathrm{b}}<-1.9 \mathrm{~V}$ (almost zero differential conductance) which corresponds to the charging region in the floating gate operation mode. The Coulomb interaction of QD localized charges with the QW results in the low conductive state with almost zero conductance. The current increase at $3.8 \mathrm{~V}$ is attributed to a discharging of the QD. We observe that the voltage ranges for charging and discharging differ from sample to sample because of varying geometries and quantum dot positions leading to small deviations in the current-voltage characteristic and pinched hysteresis loop.

Fig. 2(a) shows current-voltage-characteristics conducted in the memristive operation mode for different $V_{\mathrm{bm}}$. No pinched hysteresis loop is observed above $-1.8 \mathrm{~V}$. For smaller $V_{\mathrm{bm}}(-2.2$ and $-3.2 \mathrm{~V})$, two distinguishable conductive states (low and high) around zero bias are clearly evident. The widths of the low conductive states are 0.1 and $1.7 \mathrm{~V}$. They can be related to the voltage $V_{\mathrm{tu}}$ of the currentonset in the floating gate operation mode with $V_{\mathrm{th}} \approx 2 V_{\mathrm{tu}}$.
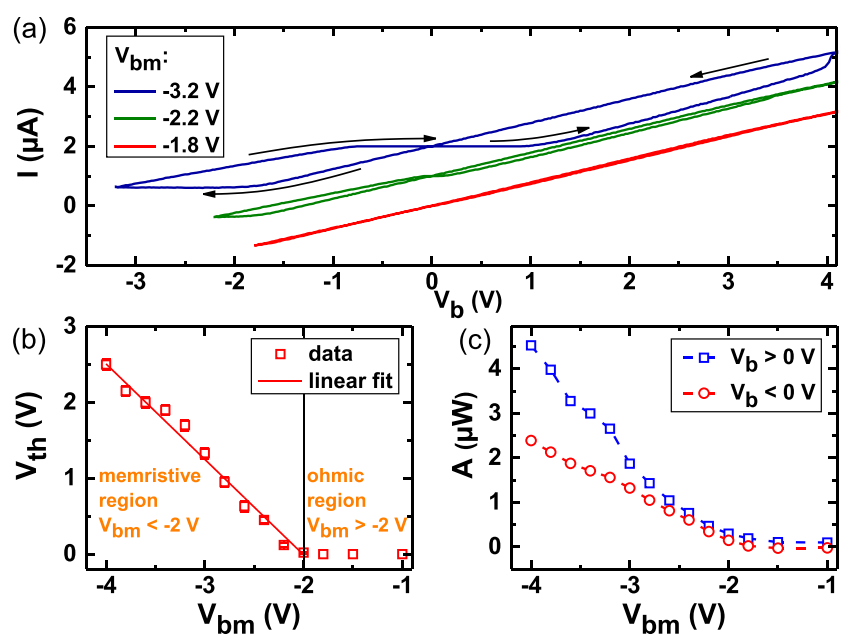

FIG. 2. (a) $I-V_{\mathrm{b}}$-characteristic of the memristive operation mode for three different minimum bias voltages $V_{\mathrm{bm}}=-1.8,-2.2$, and $-3.2 \mathrm{~V}$. The pinched hysteresis loop is observed only when the QD is charged and discharged during the sweep cycle. No hysteresis is observed for $V_{\mathrm{bm}}=-1.8 \mathrm{~V}$. For clarity, the curves are offset by $1 \mu \mathrm{A}$. (b) Width $V_{\mathrm{th}}$ versus $V_{\mathrm{bm}}$. For $V_{\mathrm{bm}}<-2.0 \mathrm{~V}$, the width increases almost linearly. Above $V_{\mathrm{bm}}>-2 \mathrm{~V}$, the number of localized charges remains constant during the sweep cycle. (c) Area $A$ enclosed by the hysteresis loop versus $V_{\mathrm{bm}}$. The area is displayed for positive and negative bias voltages. $A$ increases linearly below but is almost zero above $-2.0 \mathrm{~V}$.

Fig. 2(b) shows the plateau width versus $V_{\mathrm{bm}}$. It remains zero above (ohmic response) and increases almost linearly below $-2.0 \mathrm{~V}$ (memristive response). We attribute this linear shift to the threshold voltage shift $\Delta V_{\mathrm{tu}}=\Delta n q / C_{\text {eff }}$ of a floating gate transistor in close vicinity to a QD. ${ }^{22}$ Here, $\Delta n$ is the shift of the number of localized charges, $q$ is the elementary charge, and $C_{\text {eff }}$ accounts for the effective capacitive coupling between the QD, the 2DEG, and the gate. In the memristive operation mode, charging of the QD occurs for $V_{\mathrm{b}}<-1.9 \mathrm{~V}$ and thus $\Delta n$ is determined by $\Delta n=\left(-1.9 \mathrm{~V}-V_{\mathrm{bm}}\right) / \Delta V$ with $\Delta V$ being the voltage difference to charge the QD with one additional electron. $\Delta n$ is only defined for $V_{\mathrm{bm}} \leq 1.9 \mathrm{~V}$. For larger $V_{\mathrm{bm}}$, the QD is not charged and we observe linear current-voltage-characteristics (ohmic response). Since $V_{\mathrm{th}} \approx 2 V_{\mathrm{tu}}$, the width of the low conductive state is

$$
V_{t h} \approx \frac{2 \mathrm{q}}{\Delta V C_{e f f}}\left(-1.9 V-V_{b m}\right) .
$$

The data in Fig. 2(b) were fitted according to Eq. (1) with a shift of $\Delta V \times C_{\text {eff }} \approx 0.28 \times 10^{-18} \mathrm{C}$. The memristive operation mode can also be analyzed by means of the area $A$, which is enclosed by the pinched hysteresis loop. Fig. 2(c) shows $A$ for positive and negative bias voltages versus $V_{\mathrm{bm}}$. Similar to the plateau width, $A$ is almost zero above but increases linearly below $-2.0 \mathrm{~V}$. Both $V_{\text {th }}$ and $A$ depend on the shape of the pinched hysteresis loop, which correlates to the constitutive relation of the memristor (ideal memristor: $f(\varphi, q)=0$ with flux $\varphi){ }^{24}$ Hence, different realizations of memristors result in different shapes and the area is a characteristic parameter of the memristive operation mode.

In Fig. 3(a), current-voltage-characteristics of the memristive operation mode are plotted for different temperatures $T=4.2,145$, and $240 \mathrm{~K}$. The bias voltage was swept from $-3.5 \mathrm{~V}$ to $4.0 \mathrm{~V}$ and back. Well defined pinched hysteresis 

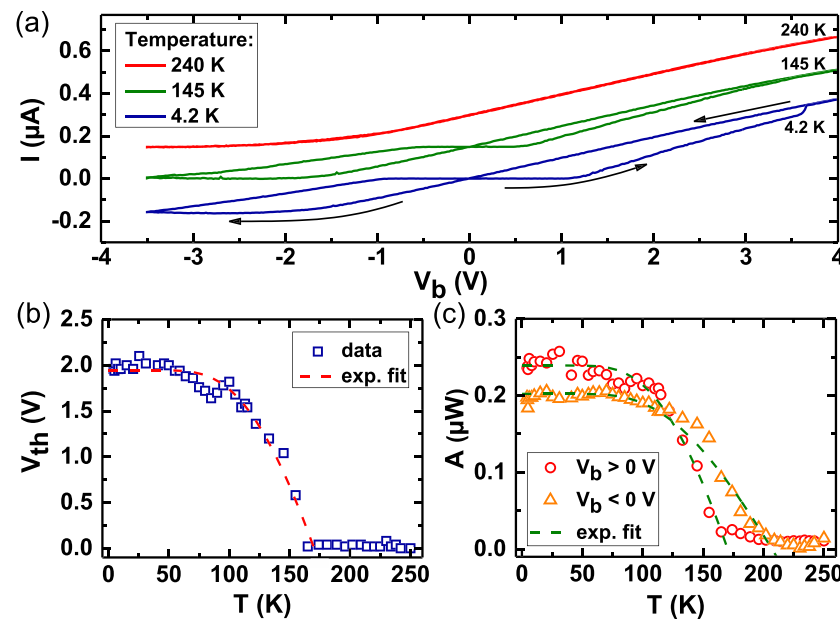

FIG. 3. (a) Current-voltage characteristics of the memristive switching mode for temperatures of $4.2,145$, and $240 \mathrm{~K}$. The pinched hysteresis loop is profound for 4.2 and $145 \mathrm{~K}$, with its two clearly distinguishable conductive states around zero bias. No pinched hysteresis loop is observed for $\mathrm{T}=240 \mathrm{~K}$. The curves are offset by $0.15 \mu \mathrm{A}$. (b) Plateau width of the low conductive state versus temperature. The maximum operating temperature is found to be $165 \mathrm{~K}$, as obtained from the exponential fit function after Eq. (4). No low conductive state is observed for higher temperatures. (c) Area of the hysteresis for positive and negative voltages versus temperature. The exponential fit functions are based on Eq. (4).

loops are observed for the temperatures ranging between 4.2 and $145 \mathrm{~K}$. For $240 \mathrm{~K}$, the currents during the up- and downsweep directions cannot be distinguished, thus $A=0$. The width of the low conductive state around zero bias as well as the area of the hysteresis loop versus the temperature are shown in Figs. 3(b) and 3(c), respectively. $V_{\text {th }}$ and $A$ remain almost constant below, but are lowered for temperatures above $50 \mathrm{~K}$. The reductions of $V_{\text {th }}$ and $A$ are associated with thermally activated discharging processes of the QD. ${ }^{21,25}$ The number of localized charges $n$ on the QD is given by the rate equation ${ }^{26}$

$$
\frac{d n}{d t}=\Gamma_{c h a}-n \Gamma_{d i s}
$$

$\Gamma_{\text {cha }}$ and $\Gamma_{\text {dis }}$ are the rates of thermally assisted charging and discharging processes, respectively. These rates can be examined using an Arrhenius type escape rate $^{27}$

$$
\Gamma=\Gamma_{0} \exp \left[-\frac{E_{\alpha}}{k T}\right] .
$$

Here, $E_{\alpha}$ are activation energies with $\alpha=$ cha, dis for the charging and discharging processes, respectively, and $k$ is the Boltzmann constant. From the steady state solution $(\mathrm{d} n /$ $\mathrm{d} t=0$ ), we obtain $V_{\mathrm{th}}$, which is directly proportional to the number $n$ of charges on the QD, as

$$
V_{t h}(T)=V_{0} \exp \left[-\frac{E_{c h a}-E_{d i s}}{k T}\right]+V_{t h}(T=0 \mathrm{~K}) .
$$

The data in Fig. 3(b) have been fitted according to Eq. (4). The fitting parameters are $V_{0}=-47.1 \mathrm{~V}, \quad V_{\mathrm{th}}(T=0 \mathrm{~K})$ $=1.9 \mathrm{~V}$, and $\Delta E=E_{\text {cha }}-E_{\text {dis }}=46.8 \mathrm{meV} . \Delta E$ represents the energetic distance between the electrochemical potentials of the 2DEG with activation energy $E_{\text {cha }}$ and the QD with activation energy $E_{\text {dis }}$. The areas of the pinched hysteresis loops in Fig. 3(c) are also fitted according to Eq. (4). The parameters are $A_{0}=-5.78 \mu \mathrm{W}, A_{\mathrm{th}}(T=0 \mathrm{~K})=0.24 \mu \mathrm{W}$ and $A_{0}=-2.78 \mu \mathrm{W}, A_{\mathrm{th}}(T=0 \mathrm{~K})=0.20 \mu \mathrm{W}$ for $V_{\mathrm{b}}>0 \mathrm{~V}$ and $\mathrm{V}_{\mathrm{b}}<0 \mathrm{~V}$, respectively. The difference of the activation energies was kept constant with $\Delta E=46.8 \mathrm{meV}$. For positive voltages, the area vanishes for a temperature of $170 \mathrm{~K}$, whereas it is still present for temperatures up to $210 \mathrm{~K}$ for negative voltages. The low temperature current-voltage characteristic and pinched hysteresis loop of the device is independent and insensitive to temperature cycles.

We also analyzed the conductance change in the memristive operation mode when applying rectangular voltage pulses as shown in Fig. 4(a). These pulses mimic the input from a pre- and a post-synaptic neuron. The post-synaptic pulses with amplitude $V_{\text {po }}$ were applied to the QW and the pre-synaptic pulses with amplitude $V_{\mathrm{pr}}$ to the QW and the gates (see Fig. 4(b)). The pulses had a width of $10 \mathrm{~ms}$ and the time difference $\Delta t$ was varied. After each pulse, the conductance $G$ was determined with a read-out pulse (dashed line). Fig. 4(c) depicts the voltage difference of the pulses. Discharging of the QD can occur during the red highlighted voltage range when the difference of both pulses exceeds the threshold voltage for discharging. The conductance versus the pulse number for amplitudes $V_{\mathrm{pr}}=3.8$ and $V_{\mathrm{po}}=-0.8 \mathrm{~V}$
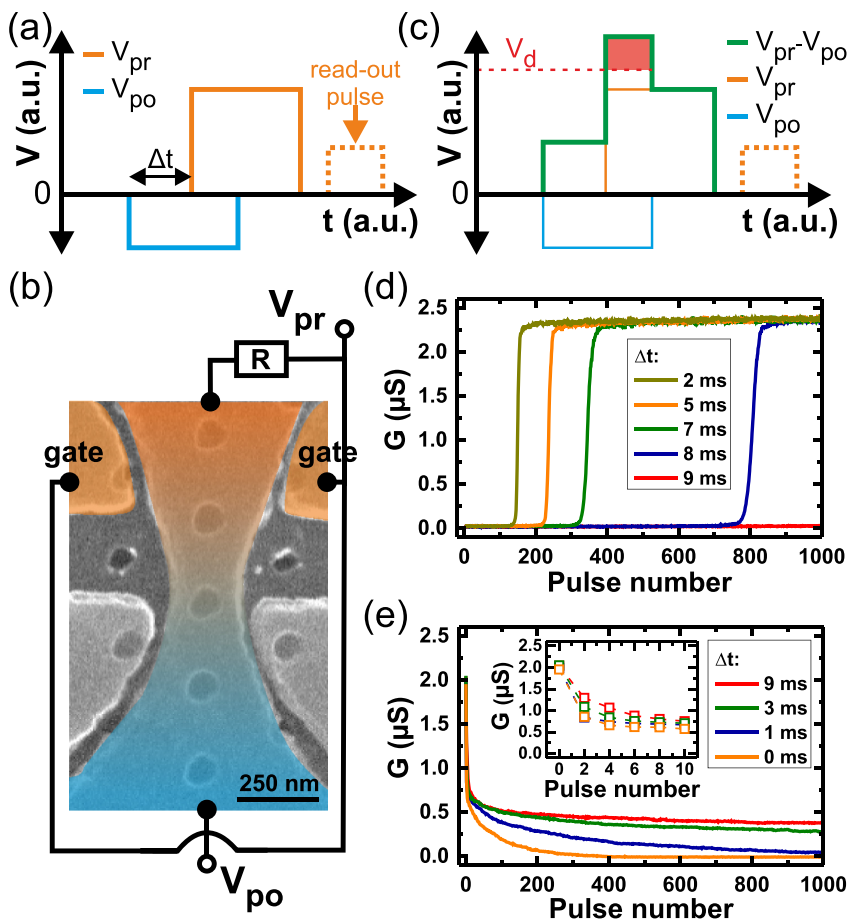

FIG. 4. (a) Time trace of the pre- and post-synaptic voltage pulses which are applied to the source and the drain side, respectively. A read-out pulse with amplitude $1.2 \mathrm{~V}$ follows the pulses to determine $G$. (b) Circuit diagram when applying pre- and post-synaptic pulses to the two terminals of the memristor. (c) Difference of the post- and pre-synaptic pulses shown in (a) versus time. During the red shaded time interval, the voltage difference exceeds the threshold voltage for discharging $V_{\mathrm{d}}$. (d) Conductance $G$ versus pulse number. The pulses shown in (a) are applied to the memristor and the conductance can be potentiated. Depending on $\Delta t$, the potentiation occurs after different pulse numbers. (e) $G$ versus pulse number for a depression of the conductance. For smaller $\Delta t$, less pulses are needed to decrease $G$ to zero. The inset shows the conductance after the first pulses in more detail. 
is presented in Fig. 4(d). Initially, the QD was charged and the conductance was almost zero. $G$ remains unaltered when applying 1000 pulses with a time difference of $9 \mathrm{~ms}$. Lowering $\Delta t$ to $8 \mathrm{~ms}$ results in an increase after 780 pulses and the conductance saturates at $2.4 \mu \mathrm{S}$. This saturation is attributed to a completely discharged QD. Less pulses are needed to discharge the QD when the time difference is reduced. The amplitudes $-2.4 \mathrm{~V}$ and $0.8 \mathrm{~V}$ of the pre- and post-synaptic pulses, respectively, are used to decrease the conductance of an initially charged QD. The data are shown in Fig. 4(e). For different $\Delta t$, the conductance is reduced by a large amount with only a few pulses. For smaller $\Delta t$, the change of $G$ is larger (see inset of Fig. 4(e)). The decreasing conductance is attributed to a charging of the QD with electrons, which can be controlled with the time difference between the pre- and post-synaptic pulses.

In summary, we have presented a quantum dot floating gate transistor featuring a memristive operation mode. Sweeping closed voltage cycles allows to charge and discharge QDs in close vicinity of a 2DEG, resulting in a pinched hysteresis loop with two clearly distinguishable conductive states around zero bias. The memristive operation mode is observed up to temperatures of $165 \mathrm{~K}$. The conductance of the device can be controlled by the charging voltage or the time difference between rectangular voltage pulses applied directly to the source and drain contact. The presented QD floating gate transistor with its memristive operation mode may be considered to realize artificial synapses in future non-von Neumann computer architectures.

The authors gratefully acknowledge financial support from the European Union (FPVII (2007-2013) under Grant Agreement No. 318287 Landauer) as well as the state of Bavaria.

${ }^{1}$ L. O. Chua, IEEE Trans. Circuit Theory 18, 507-519 (1971).

${ }^{2}$ L. O. Chua and S. M. Kang, Proc. IEEE 64, 209-223 (1976).
${ }^{3}$ D. B. Strukov, G. S. Snider, D. R. Stewart, and S. R. Williams, Nature 453, 80-83 (2008).

${ }^{4}$ A. Beck, J. G. Bednorz, Ch. Gerber, C. Rossel, and D. Widmer, Appl. Phys. Lett. 77, 139-141 (2000).

${ }^{5}$ K. Szot, W. Speier, G. Bihlmayer, and R. Waser, Nat. Mater. 5, 312-320 (2006).

${ }^{6}$ A. Sawa, T. Fujii, M. Kawasaki, and Y. Tokura, Appl. Phys. Lett. 88, 232112 (2006).

${ }^{7}$ M. N. Kozicki, M. Park, and M. Mitkova, IEEE Trans. Nanotechnol. 4, 331-338 (2005).

${ }^{8}$ K. Terabe, T. Hasegawa, T. Nakayama, and M. Aono, Nature 433, 47-50 (2005).

${ }^{9}$ Y. V. Pershin and M. Di Ventra, Phys. Rev. B 78, 113309 (2008).

${ }^{10}$ M. Ziegler, M. Oberländer, D. Schroeder, W. H. Krautschneider, and H. Kohlstedt, Appl. Phys. Lett. 101, 263504 (2012).

${ }^{11}$ C. Riggert, M. Ziegler, D. Schroeder, W. H. Krautschneider, and H. Kohlstedt, Semicond. Sci. Technol. 29, 104011 (2014).

${ }^{12}$ S. H. Jo, T. Chang, I. Ebong, B. B. Bhadviya, P. Mazumder, and W. Lu, Nano Lett. 10, 1297-1301 (2010).

${ }^{13}$ B. Linares-Barranco and T. Serrano-Gotarredona, available from Nature Precedings, see hdl:10101/npre.2009.3010.1, 2009.

${ }^{14}$ S. Song, K. D. Miller, and L. F. Abbott, Nat. Neurosci. 3, 919-926 (2000).

${ }^{15}$ G.-Q. Bi and M.-M. Poo, J. Neurosci. 18, 10464-10472 (1998).

${ }^{16}$ R. C. Malenka and R. A. Nicoll, Science 285, 1870-1874 (1999).

${ }^{17}$ T. V. P. Bliss and G. L. Collingridge, Nature 361, 31-39 (1993).

${ }^{18}$ P. A. Merolla, J. V. Arthur, R. Alvarez-Icaza, A. S. Cassidy, J. Sawada, F. Akopyan, B. L. Jackson, N. Imam, C. Guo, Y. Nakamura et al., Science 345, 668-673 (2014).

${ }^{19}$ C. Schneider, A. Huggenberger, T. Sünner, T. Heindel, M. Strauß, S. Göpfert, P. Weinmann, S. Reitzenstein, L. Worschech, M. Kamp, S. Höfling, and A. Forchel, Nanotechnology 20, 434012 (2009).

${ }^{20}$ P. Maier, F. Hartmann, M. Emmerling, C. Schneider, S. Höfling, M. Kamp, and L. Worschech, Appl. Phys. Lett. 105, 053502 (2014).

${ }^{21}$ S. Göpfert, L. Worschech, S. Lingemann, C. Schneider, D. Press, S. Höfling, and A. Forchel, Appl. Phys. Lett. 97, 222112 (2010).

${ }^{22}$ L. Guo, E. Leobandung, and S. Y. Chou, Science 275, 649-651 (1997).

${ }^{23}$ C. R. Müller, L. Worschech, and A. Forchel, Phys. Rev. B 79, 205307 (2009).

${ }^{24}$ S. P. Adhikari, M. P. Sah, H. Kim, and L. O. Chua, IEEE Trans. Circuits Syst. I 60, 3008-3021 (2013).

${ }^{25}$ A. Schliemann, L. Worschech, S. Reitzenstein, S. Kaiser, and A. Forchel, Appl. Phys. Lett. 81, 2115 (2002).

${ }^{26}$ W.-H. Chang, T. M. Hsu, C. C. Huang, S. L. Hsu, C. Y. Lai, N. T. Yeh, T. E. Nee, and J.-I. Chyi, Phys. Rev. B 62, 6959-6962 (2000).

${ }^{27}$ P. Hänggi, P. Talkner, and M. Borkovec, Rev. Mod. Phys. 62, 251-341 (1990). 